\title{
On fixed points of permutations
}

\author{
Persi Diaconis · Jason Fulman • Robert Guralnick
}

Received: 20 August 2007 / Accepted: 3 April 2008 / Published online: 22 April 2008

(C) Springer Science+Business Media, LLC 2008

\begin{abstract}
The number of fixed points of a random permutation of $\{1,2, \ldots, n\}$ has a limiting Poisson distribution. We seek a generalization, looking at other actions of the symmetric group. Restricting attention to primitive actions, a complete classification of the limiting distributions is given. For most examples, they are trivial - almost every permutation has no fixed points. For the usual action of the symmetric group on $k$-sets of $\{1,2, \ldots, n\}$, the limit is a polynomial in independent Poisson variables. This exhausts all cases. We obtain asymptotic estimates in some examples, and give a survey of related results.
\end{abstract}

Keywords Fixed point · Derangement · Primitive action · O’Nan-Scott theorem

\section{Introduction}

One of the oldest theorems in probability theory is the Montmort (1708) limit theorem for the number of fixed points of a random permutation of $\{1,2, \ldots, n\}$. Let $S_{n}$ be the symmetric group. For an element $w \in S_{n}$, let $F(w)=|\{i: w(i)=i\}|$. Montmort [58]

This paper is dedicated to the life and work of our colleague Manfred Schocker.

We thank Peter Cameron for his help. Diaconis was supported by NSF grant DMS-0505673. Fulman received funding from NSA grant H98230-05-1-0031 and NSF grant DMS-0503901. Guralnick was supported by NSF grant DMS-0653873. This research was facilitated by a Chaire d'Excellence grant to the University of Nice Sophia-Antipolis.

\section{P. Diaconis}

Department of Mathematics and Statistics, Stanford University, Stanford, CA 94305, USA

J. Fulman $(\bowtie) \cdot$ R. Guralnick

Department of Mathematics, University of Southern California, Los Angeles, CA 90089-2532, USA

e-mail: fulman@usc.edu

R. Guralnick

e-mail: guralnic@usc.edu 
proved that

$$
\frac{|\{w: F(w)=j\}|}{n !} \rightarrow \frac{1}{e} \frac{1}{j !}
$$

for $j$ fixed as $n$ tends to infinity. The limit theorem (1.1) has had many refinements and variations. See Takács [68] for its history, Chapter 4 of Barbour, Holst, Janson [7] or Chatterjee, Diaconis, Meckes [18] for modern versions.

The limiting distribution $P_{\lambda}(j)=e^{-\lambda} \lambda^{j} / j$ ! (in (1.1) $\lambda=1$ ) is the Poisson distribution of "the law of small numbers". Its occurrence in many other parts of probability (see e.g. Aldous [1]) suggests that we seek generalizations of (1.1), searching for new limit laws.

In the present paper we look at other finite sets on which $S_{n}$ acts. It seems natural to restrict to transitive actions - otherwise, things break up into orbits in a transparent way. It is also natural to restrict to primitive actions. Here $S_{n}$ acts primitively on the finite set $\Omega$ if we cannot partition $\Omega$ into disjoint blocks $\Delta_{1}, \Delta_{2}, \ldots, \Delta_{h}$ where $S_{n}$ permutes the blocks (if $\Delta_{i}^{w} \cap \Delta_{j} \neq \phi$ then $\Delta_{i}^{w}=\Delta_{j}$ ). The familiar wreath products which permute within blocks and between blocks give examples of imprimitive actions.

The primitive actions of $S_{n}$ have been classified in the O'Nan-Scott theorem. We describe this carefully in Section 2. For the study of fixed points most of the cases can be handled by a marvelous theorem of Luczak and Pyber [57]. This shows that, except for the action of $S_{n}$ on $k$-sets of an $n$-set, almost all permutations have no fixed points (we say $w$ is a derangement). This result is explained in Section 3. For $S_{n}$ acting on $k$-sets, one can assume that $k<n / 2$. Then there is a nontrivial limit if and only if $k$ stays fixed as $n$ tends to infinity. In these cases, the limit is shown to be an explicit polynomial in independent Poisson random variables. This is the main content of Section 4. Section 5 works out precise asymptotics for the distribution of fixed points in the action of $S_{n}$ on matchings. Section 6 considers more general imprimitive subgroups. Section 7 proves that the proportion of elements of $S_{n}$ which belong to a primitive subgroup not containing $A_{n}$ is at most $O\left(n^{-2 / 3+\alpha}\right)$ for any $\alpha>$ 0 ; this improves on the bound of Luczak and Pyber [57]. Finally, Section 8 surveys related results (including analogs of our main results for finite classical groups) and applications of the distribution of fixed points and derangements.

\subsection{Probability notation and inequalities}

We conclude this introduction with basic probabilistic notation and inequalities used throughout. Useful background for this material is in [33] or [32]. If $P$ is a probability measure on the finite or countable set $\Omega$ and $T: \Omega \rightarrow \mathbb{R}$ is a function, we write $P(T=$ $t):=\sum_{\{\omega \in \Omega \mid T(\omega)=t\}} P(\omega)$. The mean of $T$ is denoted $E(T):=\sum_{\omega \in \Omega} T(\omega) P(\omega)$. The variance of $T$ is denoted $\operatorname{Var}(T):=E\left(T^{2}\right)-E(T)^{2}$. Usually, $\Omega$ is a finite group $G$ and $P(\omega)=1 /|G|$. For $\lambda \geq 0$, define the Poisson probability measure with parameter $\lambda$ as $P(j)=e^{-\lambda} \lambda^{j} / j$ ! on $\Omega=\mathbb{N}=\{0,1, \ldots\}$. Define a probability measure on $\mathbb{N}^{n}$ by the equation $P\left(j_{1}, \ldots, j_{n}\right)=\prod_{k=1}^{n} e^{-\lambda_{k}} \lambda_{k}^{j_{k}} /\left(j_{k}\right)$ !. This measure makes the coordinate functions $X_{i}\left(j_{1}, \ldots, j_{n}\right)=j_{i}$ independent in the sense of the equality $P\left(X_{1}=j_{1}, \ldots, X_{n}=j_{n}\right)=\prod P\left(X_{i}=j_{i}\right)$. The $X_{i}$ are called independent Poisson random variables with parameters $\lambda_{i}$. 
If a finite group $G$ acts on $\Omega$ with $F(w)$ the number of fixed points of $w$, the "lemma that is not Burnside's" implies that

$$
\begin{aligned}
E(F(w)) & =\# \text { orbits of } G \text { on } \Omega, \\
E\left(F^{2}(w)\right) & =\# \text { orbits of } G \text { on } \Omega \times \Omega=\operatorname{rank}:=r .
\end{aligned}
$$

If $G$ is transitive on $\Omega$ with isotropy group $H$, then the rank is also the number of orbits of $H$ on $\Omega$ and so equal to the number of $H-H$ double cosets in $G$.

Thus for transitive actions

$$
E(F(w))=1, \quad \operatorname{Var}(F(w))=\operatorname{rank}-1 .
$$

In most of our examples $P(F(w)=0) \rightarrow 1$ but because of (1.2), this cannot be seen by moment methods. The standard second moment method (Durrett [32], page 16) says that if $X$ is a non-negative random variable such that $a<E X$ and $E X^{2}<\infty$, then $P(X>a) \geq(E X-a)^{2} / E\left(X^{2}\right)$. Specializing to our case and taking $a=0$ gives that, $P(F(w)>0) \geq 1 / r$; therefore $P(F(w)=0) \leq 1-1 / r$. This shows that the convergence to 1 cannot be too rapid. It also shows that $P(F(w)=0)$ tends to 1 implies that the rank tends to infinity. Indeed, for primitive actions of symmetric and alternating groups, this is also a sufficient condition - see Theorem 3.4.

There is also an elementary lower bound $P(F(w)=0) \geq(r-1) / n$. Even the simplest instance of this lower bound (i.e. $1 / n)$ was only observed in 1992 in [17]. We reproduce the simple proof from [46] in Section 3.

While preparing this paper, we came across a remarkable paper of Manfred Schocker which makes extensive use of the classical derangement numbers. In particular, he gives formulae and development for the derangement characters $\chi_{n, k}$. These are the characters arising from the linear span of indicator functions of all permutations in $S_{n}$ with exactly $k$ fixed points. The main tool is a novel commutative, semisimple, $n$-dimensional subalgebra of Solomon's descent algebra, given with explicit idempotents. While it would take us too far afield to develop this here, we note that Schocker's algebra appears earlier in the analysis of "top to random" shuffles (Section 4 of [24]).

\section{O'Nan-Scott theorem}

Let $G$ act transitively on the finite set $\Omega$. By standard theory we may represent $\Omega$ as the left coset space $G / G_{\alpha}$ with any fixed $\alpha \in \Omega$. Here $G_{\alpha}=\left\{w: \alpha^{w}=\alpha\right\}$ with the action being left multiplication on the cosets. Further (Passman [62, 3.4]) the action of $G$ on $\Omega$ is primitive if and only if the isotropy group $G_{\alpha}$ is maximal. Thus, classifying primitive actions of $G$ is the same problem as classifying maximal subgroups $H$ of $G$.

The O'Nan-Scott theorem classifies maximal subgroups of $A_{n}$ and $S_{n}$ up to determining the almost simple primitive groups of degree $n$. Definitions of terms used in the theorem are given in the remarks and examples following its statement.

Theorem 2.1 [O'Nan-Scott] Let $H$ be a maximal subgroup of $G=A_{n}$ or $S_{n}$. Then, one of the following three cases holds: 
I: $\quad H$ acts primitively as a subgroup of $S_{n}$ (primitive case),

II: $H=\left(S_{a}<S_{b}\right) \cap G$ (wreath product), $n=a \cdot b,|\Omega|=\frac{n !}{(a !)^{b} \cdot b !}$ (imprimitive case), or

III: $H=\left(S_{k} \times S_{n-k}\right) \cap G,|\Omega|=\left(\begin{array}{l}n \\ k\end{array}\right)$ with $1 \leq k<n / 2$ (intransitive case).

Further, in case I, one of the following holds:

Ia: $H$ is almost simple,

Ib: $H$ is diagonal,

Ic: $H$ preserves product structure, or

Id: $H$ is affine.

Remarks and examples:

(1) Note that in cases I, II, III, the modifiers 'primitive', 'imprimitive', 'intransitive' apply to $H$ in its action on $\{1, \ldots, n\}$. Since $H$ is maximal in $G, \Omega \cong G / H$ is a primitive $G$-set. We present an example and suitable additional definitions for each case.

(2) In case III, $\Omega$ is the $k$-sets of $\{1,2, \ldots, n\}$ with the obvious action of $S_{n}$. This case is discussed extensively in Section 4 below.

(3) In case II, take $n$ even with $a=2, b=n / 2$. We may identify $\Omega$ with the set of perfect matchings on $n$ points - partitions of $n$ into $n / 2$ two-element subsets where order within a subset or among subsets does not matter. For example if $n=6,\{1,2\}\{3,4\}\{5,6\}$ is a perfect matching. For this case, $|\Omega|=\frac{n !}{2^{n / 2}(n / 2) !}=$ $(n-1)(n-3) \cdots(1)$. Careful asymptotics for this case are developed in Section 5. More general imprimitive subgroups are considered in Section 6.

(4) While every maximal subgroup of $A_{n}$ or $S_{n}$ falls into one of the categories of the O'Nan-Scott theorem, not every such subgroup is maximal. A complete list of the exceptional examples is in Liebeck, Praeger and Saxl [54]. This depends heavily on the classification of finite simple groups.

(5) In case Ia, $H$ is almost simple if for some non-Abelian simple group $G, G \leq H \leq$ $\operatorname{Aut}(G)$. For example, fix $1<k<\frac{m}{2}$. Let $n=\left(\begin{array}{c}m \\ k\end{array}\right)$. Let $S_{n}$ be all $n$ ! permutations of the $k$ sets of $\{1,2, \ldots, m\}$. Take $S_{m} \leq S_{n}$ acting on the $k$-sets in the usual way. For $m \geq 5, S_{m}$ is almost simple and primitive. Here $\Omega=S_{n} / S_{m}$ does not have a simple combinatorial description, but this example is crucial and the $k=2$ case will be analyzed in Section 7.

Let $\tau \in S_{m}$ be a transposition. Then $\tau$ moves precisely $2\left(\begin{array}{c}m-2 \\ k-1\end{array}\right) k$-sets. Thus, $S_{m}$ embeds in $A_{n}$ if and only if $\left(\begin{array}{c}m-2 \\ k-1\end{array}\right)$ is even. Indeed for most primitive embeddings of $S_{m}$ into $S_{n}$, the image is contained in $A_{n}$ [60].

It is not difficult to see that the image of $S_{m}$ is maximal in either $A_{n}$ or $S_{n}$. This follows from the general result in [54]. It also follows from the classification of primitive groups containing a non-trivial element fixing at least $n / 2$ points [45].

Similar examples can be constructed by looking at the action of $P \Gamma L_{d}(q)$ on $k$-spaces (recall that $P \Gamma L_{d}(q)$ is the projective group of all semilinear transformations of a $d$ dimensional vector space over $\mathbb{F}_{q}$ ). All of these are covered by case $I a$.

(6) To describe case $\mathrm{Ib}$, take $G_{1}$ a non-Abelian simple group and $k \geq 2$. Set $\Omega=$ $G_{1}^{k} / D$ with $D=\{(g, g, \ldots g)\}_{g \in G_{1}}$ the diagonal subgroup. Clearly $G_{1}^{k}$ acts on $\Omega$. 
Further Aut $\left(G_{1}\right)$ acts on $G_{1}^{k}$ by applying the same automorphism on each coordinate. This normalizes $D$ and so also acts on $\Omega$. Finally, $S_{k}$ acts on $G_{1}^{k}$ by permuting coordinates. Since it even centralizes $D$, it also acts on $\Omega$. Take $H$ to be the group generated by these three types of permutations (i.e. $G_{1}^{k}$, Aut $\left.\left(G_{1}\right), S_{k}\right)$. The group $H$ has $G_{1}^{k}$ as a normal subgroup with quotient isomorphic to $\operatorname{Out}\left(G_{1}\right) \times S_{k}$. This may or may not be a split extension; it splits if and only if the extension $1 \rightarrow G_{1} \rightarrow \operatorname{Aut}\left(G_{1}\right) \rightarrow \operatorname{Out}\left(G_{1}\right) \rightarrow 1$ splits.

We remark that $H \cap A_{n}$ is always maximal in $A_{n}$ [54]. It can be subtle to determine whether or not $H \leq A_{n}$.

Here is a specific example. Take $S=G_{1}$ for $m \geq 8$ and $k=2$. Then $\operatorname{Out}\left(A_{m}\right)=C_{2}$ and so $H=\left\langle A_{m} \times A_{m}, \tau,(s, s)\right\rangle$ where $s$ is a transposition (or any element in $S_{m}$ outside of $A_{m}$ ) and $\tau$ is the involution changing coordinates. More precisely, each coset of $D$ has a unique representative of the form $(1, x)$. We have $\left(g_{1}, g_{2}\right)(1, x) D=\left(g_{1}, g_{2} x\right) D=\left(1, g_{2} x g_{1}^{-1}\right) D$. The action of $\tau \in C_{2}$ takes $(1, x) \rightarrow\left(1, x^{-1}\right)$ and the action of $(s, s) \in \operatorname{Out}\left(A_{m}\right)$ takes $(1, x)$ to $\left(1, s x s^{-1}\right)$.

We first show that if $m \geq 8$, then $H$ is contained in $\operatorname{Alt}(\Omega)$. Clearly $A_{m} \times A_{m}$ is contained in $\operatorname{Alt}(\Omega)$. Observe that $(s, s)$ is contained in $\operatorname{Alt}(\Omega)$. Indeed, taking $s$ to be a transposition, the number of fixed points of $(s, s)$ is the size of the centralizer of $s$ in $A_{m}$ which is $\left|S_{m-2}\right|$, and so $\frac{m !}{2}-(m-2)$ ! points are moved and this is divisible by 4 since $m \geq 8$. To see that $\tau$ is contained in $\operatorname{Alt}(\Omega)$ for $m \geq 8$, note that the number of fixed points of $\tau$ is the number of involutions (including the identity) in $A_{m}$, so it is sufficient to show that $\frac{m !}{2}$ minus this number is a multiple of 4 . This follows from the next proposition, which is of independent combinatorial interest.

Note in the example, the extension is split for $m \neq 6$, but it is not split for $m=6$ (because $\operatorname{Aut}\left(A_{6}\right)$ is not split over $\left.A_{6}\right)$.

Proposition 2.2 Suppose that $m \geq 8$. Then the number of involutions in $A_{m}$ and the number of involutions in $S_{m}$ are multiples of 4 .

Proof Let $a(m)$ be the number of involutions in $A_{m}$ (including the identity). Let $b(m)$ be the number of involutions in $S_{m}-A_{m}$. It suffices to show that $a(m)=b(m)=0$ $\bmod 4$. For $n=8,9$ we compute directly. For $n>9$, we observe that

$$
a(n)=a(n-1)+(n-1) b(n-2)
$$

and

$$
b(n)=b(n-1)+(n-1) a(n-2)
$$

(because an involution either fixes 1 giving the first term or swaps 1 with $j>1$, giving rise to the second term). The result follows by induction.

Having verified that $H$ is contained in $\operatorname{Alt}(\Omega)$ for $m \geq 8$, maximality in $\operatorname{Alt}(\Omega)$ now follows from Liebeck-Praeger-Saxl [54].

(7) In case Ic, $H$ preserves a product structure. Let $\Gamma=\{1, \ldots, m\}, \Delta=\{1, \ldots, t\}$, and let $\Omega$ be the $t$-fold Cartesian product of $\Gamma$. If $C$ is a permutation group on $\Gamma$ 
and $D$ is a permutation group on $\Delta$, we may define a group $H=C$ 2 $D$ by having $C$ act on the coordinates, and having $D$ permute the coordinates. Primitivity of $H$ is equivalent to $C$ acting primitively on $\Gamma$ with some non identity element having a fixed point and $D$ acting transitively on $\Delta$ (see, e.g. Cameron [15], Th. 4.5).

Since we are assuming that $H$ is maximal, we see that $H$ is the maximal subgroup preserving the product structure. Thus, $G=S_{m^{t}}, H=S_{m} 2 S_{t}$ and $\Omega$ is the t-fold Cartesian product $\{1, \cdots, m\}^{t}$. If $m>4$, then $H$ is maximal in either $S_{m^{t}}$ or $A_{m^{t}}$, and $H \cap A_{m^{t}}$ is maximal in $A_{m^{t}}$. It is easy to determine when $H$ embeds in $A_{m}$. The case $t=2$ will be analyzed in detail in Section 7. We just note that if $t=2$, then $H \leq A_{m^{2}}$ if and only if $4 \mid \mathrm{m}$.

(8) In case Id, $H$ is affine. Thus $\Omega=V$, a vector space of dimension $k$ over a field of $q$ elements ( $\operatorname{so} n=|\Omega|=q^{k}$ ) and $H$ is the semidirect product $V \cdot G L(V)$. Since we are interested only in maximal subgroups, $q$ must be prime.

Note that if $q$ is odd, then $H$ contains an $n-1$ cycle and so is not contained in $A_{n}$. If $q=2$, then for $k>2, H$ is perfect and so is contained in $A_{n}$. The maximality of $H$ in $A_{n}$ or $S_{n}$ follows by Mortimer [59] for $k>1$ and [44] if $k=1$.

(9) The proof of the O'Nan-Scott theorem is not difficult. O'Nan and Scott each presented proofs at the Santa Cruz Conference in 1979. A textbook presentation is [31]; see also the lively lecture notes of Cameron ([15], Chapter 4). The notion of generalized Fitting subgroup is quite useful in both the proof and statement of the theorem. See Kurzweil and Stellmacher [51].

There is a more delicate version which describes all primitive permutation groups. This was proved in Aschbacher-Scott [5] giving quite detailed information. A short proof of the Aschbacher-O'Nan-Scott theorem is in [42]. See also Liebeck, Praeger and Saxl [55].

\section{Derangements and rank}

The main new result of this section is Theorem 3.4, which shows that the proportion of derangements goes to 1 whenever it can.

We first state the elementary result already discussed in the introduction giving bounds for the proportion of derangements.

Theorem 3.1 Let $G$ be a finite transitive permutation group of degree $n$ and rank $r$. Then

$$
\frac{r-1}{n} \leq P(F(w)=0) \leq 1-\frac{1}{r}
$$

Proof We reproduce the simple proof from [46] for the lower bound. Let $G_{0}$ be the set of derangements. Note that $F(w) \leq n$, whence

$$
\sum_{G}(F(w)-1)(F(w)-n) \leq \sum_{G_{0}}(F(w)-1)(F(w)-n)=n\left|G_{0}\right| .
$$


On the other hand, the left hand side is equal to $|G|(r-1)$. It follows that $P(F(w)=$ $0) \geq(r-1) / n$.

As we have already noted, the upper bound follows from the standard second moment method.

The following two results are due to Luczak and Pyber. In the statement of the second result, a subgroup of $S_{n}$ is called transitive if it acts transitively on $\{1, \cdots, n\}$.

Theorem 3.2 ([57]) Let $S_{n}$ act on $\{1,2, \ldots, n\}$ as usual and let $i(n, k)$ be the number of $w \in S_{n}$ that leave some $k$-element set invariant. Then, $\frac{i(n, k)}{n !} \leq a k^{-.01}$ for an absolute constant $a$.

Theorem 3.3 ([57]) Let $t_{n}$ denote the number of elements of the symmetric group $S_{n}$ which belong to transitive subgroups different from $S_{n}$ or $A_{n}$. Then

$$
\lim _{n \rightarrow \infty} t_{n} / n !=0 \text {. }
$$

Theorem 3.2 is at the heart of the proof of Theorem 3.3. We use them both to prove the main result of this section.

Theorem 3.4 Let $G_{i}$ be a finite symmetric or alternating group of degree $n_{i}$ acting primitively on a finite set $\Omega_{i}$ of cardinality at least 3 . Assume that $n_{i} \rightarrow \infty$. Let $d_{i}$ be the proportion of $w \in G_{i}$ with no fixed points. Then the following are equivalent:

(1) $\lim _{i \rightarrow \infty} d_{i}=1$,

(2) there is no fixed $k$ with $\Omega_{i}=\left\{k\right.$ - sets of $\left.\left\{1,2, \ldots, n_{i}\right\}\right\}$ for infinitely many $i$, and

(3) the rank of $G_{i}$ acting on $\Omega_{i}$ tends to $\infty$.

Proof First we show that (2) implies (1). Let $H_{i}$ be an isotropy group for $G_{i}$ acting on $\Omega_{i}$, and recall that $d_{i}$ is the proportion of elements of $S_{n}$ not contained in any conjugate of $H_{i}$. If $H_{i}$ falls into category I or II of the O'Nan-Scott theorem, $H_{i}$ is transitive. Writing out Theorem 3.3 above more fully, Luczak-Pyber prove that

$$
\frac{\left|\bigcup_{H} H\right|}{n !} \rightarrow 0
$$

where the union is over all transitive subgroups of $S_{n}$ not equal to $S_{n}$ or $A_{n}$. Thus a randomly chosen $w \in G_{i}$ is not in any $x H_{i} x^{-1}$ if $H_{i}$ falls into category I or II.

For $H_{i}$ in category III ( $k$-sets of an $n$-set), Theorem 3.2 gives that the proportion of elements in any conjugate of $H_{i}$ is at most $a / k^{.01}$ for an absolute constant $a$. This goes to 0 as $k \rightarrow \infty$, completing the proof that (2) implies (1).

To see that (1) implies (3), note that if the rank does not go to $\infty$, then $d_{i}$ cannot approach 1 by Theorem 3.1. To see that (3) implies (2), recall that the rank of the action on $k$-sets is $k+1$.

The analog of the previous theorem does not hold for all finite simple groups, but there is a version that is true for groups over a fixed field; see Subsection 8.2. 


\section{$4 k$-sets of an $n$-set}

In this section the limiting distribution of the number of fixed points of a random permutation acting on $k$-sets of an $n$-set is determined.

Theorem 4.1 Fix $k$ and let $S_{n}$ act on $\Omega_{n, k}-$ the $k$-sets of $\{1,2, \ldots, n\}$. Let $A_{i}(w)$ be the number of $i$-cycles of $w \in S_{n}$ in its usual action on $\{1,2, \ldots, n\}$. Let $F_{k}(w)$ be the number of fixed points of $w$ acting on $\Omega_{n, k}$. Then

(1)

$$
F_{k}(w)=\sum_{|\lambda|=k} \prod_{i=1}^{k}\left(\begin{array}{c}
A_{i}(w) \\
n_{i}(\lambda)
\end{array}\right) .
$$

Here the sum is over partitions $\lambda$ of $k$ and $n_{i}(\lambda)$ is the number of parts of $\lambda$ equal to $i$.

(2) For all $n \geq 2, E\left(F_{k}\right)=1, \operatorname{Var}\left(F_{k}\right)=k$.

(3) As $n$ tends to infinity, $A_{i}(w)$ converge to independent Poisson random variables with parameters $(1 / i)$.

Proof If $w \in S_{n}$ is to fix a $k$-set, the cycles of $w$ must be grouped to partition $k$. The expression for $F_{k}$ just counts the distinct ways to do this. See the examples below. This proves part 1 . The rank of $S_{n}$ acting on $k$-sets is $k+1$, proving part 2 .

The joint limiting distribution of the $A_{i}$ is a classical result due to Goncharov [41]. In fact, letting $X_{1}, X_{2}, \cdots, X_{k}$ be independent Poisson random variables with parameters $1, \frac{1}{2}, \cdots, \frac{1}{k}$, one has from [28] that for all $n \geq \sum_{i=1}^{k} i b_{i}$,

$$
E\left(\prod_{i=1}^{k} A_{i}(w)^{b_{i}}\right)=\prod_{i=1}^{k} E\left(X_{i}^{b_{i}}\right) .
$$

Part 3 now follows from the classical method of moments. For total variation bounds see [2].

Examples. Throughout, let $X_{1}, X_{2}, \ldots, X_{k}$ be independent Poisson random variables with parameters $1, \frac{1}{2}, \frac{1}{3}, \ldots, \frac{1}{k}$ respectively. This means that

$$
P\left(X_{1}=n_{1}, \cdots, X_{k}=n_{k}\right)=\prod_{i=1}^{k} \frac{e^{-1 / i}}{i^{n_{i}} n_{i} !} .
$$

$k=1$ : This is the usual action of $S_{n}$ on $\{1,2, \ldots, n\}$ and Theorem 4.1 yields (1.1) of the introduction: In particular, for derangements

$$
P\left(F_{1}(w)=0\right) \rightarrow 1 / e \doteq .36788 .
$$

$k=2$ : Here $F_{2}(w)=\left(\begin{array}{c}A_{1}(w) \\ 2\end{array}\right)+A_{2}(w)$ and Theorem 4.1 says that $P\left(F_{2}(w)=j\right) \rightarrow P\left(\left(\begin{array}{c}X_{1} \\ 2\end{array}\right)+X_{2}=j\right)$ where $X_{1}$ is Poisson with parameter 1 and $X_{2}$ is Poisson with parameter $\frac{1}{2}$. 
In particular

$$
P\left(F_{2}(w)=0\right) \rightarrow \frac{2}{e^{3 / 2}} \doteq .44626 .
$$

$k=3:$ Here $F_{3}(w)=\left(\begin{array}{c}A_{1}(w) \\ 3\end{array}\right)+A_{1}(w) A_{2}(w)+A_{3}(w)$ and

$$
P\left(F_{3}(w)=j\right) \rightarrow P\left(\left(\begin{array}{c}
X_{1} \\
3
\end{array}\right)+X_{1} X_{2}+X_{3}=j\right) .
$$

In particular

$$
P\left(F_{3}(w)=0\right) \rightarrow \frac{1}{e^{4 / 3}}\left(1+\frac{3}{2} e^{-1 / 2}\right) \doteq .50342 .
$$

We make the following conjecture, which has also been independently stated as a problem by Cameron [16].

Conjecture: $\lim _{n \rightarrow \infty} P\left(F_{k}(w)=0\right)$ is increasing in $k$.

Using Theorem 4.1, one can prove the following result which improves, in this context, the upper bound given in Theorem 3.1.

\section{Proposition 4.2}

$$
\lim _{n \rightarrow \infty} P\left(F_{k}(w)=0\right) \leq 1-\frac{\log (k)}{k}+O\left(\frac{1}{k}\right) .
$$

Proof Clearly

$$
\begin{aligned}
& P\left(F_{k}(w)>0\right) \geq P\left(\bigcup_{j=1}^{\left\lfloor\frac{k-1}{2}\right\rfloor}\left(A_{k-j}>0 \text { and } A_{j}>0\right)\right) \\
&=1-P\left(\bigcap_{j=1}^{\left\lfloor\frac{k-1}{2}\right\rfloor} \overline{\left(A_{k-j}>0 \text { and } A_{j}>0\right)}\right) .
\end{aligned}
$$

By Theorem 4.1, this converges to

$$
1-\prod_{j=1}^{\left\lfloor\frac{k-1}{2}\right\rfloor}\left[1-\left(1-e^{-1 / j}\right)\left(1-e^{-1 /(k-j)}\right)\right] .
$$

Let $a_{j}=\left(1-e^{-1 / j}\right)$. Write the general term in the product as $e^{\log \left(1-a_{j} a_{k-j}\right)}$. Expand the $\log$ to $-a_{j} a_{k-j}+O\left(\left(a_{j} a_{k-j}\right)^{2}\right)$. Writing $a_{j}=\frac{1}{j}+O\left(\frac{1}{j^{2}}\right)$ and multiplying out, 
we must sum

$$
\sum_{j=1}^{\left\lfloor\frac{k-1}{2}\right\rfloor} \frac{1}{j} \frac{1}{k-j}, \sum_{j=1}^{\left\lfloor\frac{k-1}{2}\right\rfloor} \frac{1}{j^{2}} \frac{1}{k-j}, \sum_{j=1}^{\left\lfloor\frac{k-1}{2}\right\rfloor} \frac{1}{(k-j)^{2}} \frac{1}{k}, \sum_{j=1}^{\left\lfloor\frac{k-1}{2}\right\rfloor} \frac{1}{(k-j)^{2}} \frac{1}{j^{2}} .
$$

Writing $\frac{1}{j} \frac{1}{k-j}=\frac{1}{k}\left(\frac{1}{j}+\frac{1}{k-j}\right)$, the first sum is $\frac{\log (k)}{k}+O\left(\frac{1}{k}\right)$. The second sum is $O\left(\frac{1}{k}\right)$, the third sum is $O\left(\frac{\log (k)}{k^{2}}\right)$ and the fourth is $O\left(\frac{1}{k^{2}}\right)$. Thus $-a_{j} a_{k-j}$ summed over $1 \leq j \leq(k-1) / 2$ is $-\frac{\log (k)}{k}+O\left(\frac{1}{k}\right)$. The sum of $\left(a_{j} a_{k-j}\right)^{2}$ is of lower order by similar arguments. In all, the lower bound on $\lim _{n \rightarrow \infty} P\left(F_{k}(w)>0\right)$ is

$$
1-e^{-\frac{\log (k)}{k}+O\left(\frac{1}{k}\right)}=\frac{\log (k)}{k}+O\left(\frac{1}{k}\right) .
$$

To close this section, we give a combinatorial interpretation for the moments of the numbers $F_{k}(w)$ of Theorem 4.1 above. This involves the "top k to random" shuffle, which removes $\mathrm{k}$ cards from the top of the deck, and randomly riffles them with the other n-k cards (choosing one of the $\left(\begin{array}{l}n \\ k\end{array}\right)$ possible interleavings uniformly at random). Note that the top $\mathrm{k}$ to random shuffle is the inverse of the move $\mathrm{k}$ to front shuffle, which picks $\mathrm{k}$ cards at random and moves them (cards higher up in the deck remaining higher) to the front of the deck.

\section{Proposition 4.3}

(1) The eigenvalues of the top $k$ to random shuffle are the numbers $\left\{\frac{F_{k}(w)}{\left(\begin{array}{c}n \\ k\end{array}\right)}\right\}$, where $w$ ranges over $S_{n}$.

(2) For all values of $n, k, r$, the rth moment of the distribution of fixed $k$-sets is equal to $\left(\begin{array}{l}n \\ k\end{array}\right)^{r}$ multiplied by the chance that the top $k$ to random shuffle is at the identity after $r$ steps.

Proof Let $M$ be the transition matrix of the top k to random shuffle; this matrix is of order $n$ ! with states the possible orderings of the deck. By the sentence before the proof, the transition matrix of the move $\mathrm{k}$ to front shuffle is the transpose of $M$. Hence it has the same eigenvalues. The move $\mathrm{k}$ to front shuffle is a special case of the theory of random walk on chambers of hyperplane arrangements developed in [10]. The arrangement is the braid arrangement and one assigns weight $\frac{1}{\left(\begin{array}{c}n \\ k\end{array}\right)}$ to each of the block ordered partitions where the first block has size $k$ and the second block has size $n-k$. The result now follows from Corollary 2.2 of [10], which determined the eigenvalues of such hyperplane walks.

For the second assertion, note that $\operatorname{Tr}\left(M^{r}\right)$ (the trace of $M^{r}$ ) is equal to $n$ ! multiplied by the chance that the top $\mathrm{k}$ to random shuffle is at the identity after $\mathrm{r}$ steps. The first part gives that

$$
\operatorname{Tr}\left(M^{r}\right)=\sum_{w \in S_{n}}\left(\frac{F_{k}(w)}{\left(\begin{array}{l}
n \\
k
\end{array}\right)}\right)^{r},
$$


which implies the result.

As an example of part 2 of Proposition 4.3, the chance of being at the identity after 1 step is $\frac{1}{\left(\begin{array}{c}n \\ k\end{array}\right)}$ and the chance of being at the identity after 2 steps is $\frac{k+1}{\left(\begin{array}{c}n \\ k\end{array}\right)^{2}}$, giving another proof that $E\left(F_{k}(w)\right)=1$ and $E\left(F_{k}^{2}(w)\right)=k+1$.

\section{Remark}

(1) As in the proof of Proposition 4.1, the moments of $F_{k}(w)$ can be expressed exactly in terms of the moments of Poisson random variables, provided that $n$ is sufficiently large.

(2) There is a random walk on the irreducible representations of $S_{n}$ which has the same eigenvalues as the top $\mathrm{k}$ to random walk, but with different multiplicities. Unlike the top $\mathrm{k}$ to random walk, this walk is reversible with respect to its stationary distribution, so that spectral techniques (and hence information about the distribution of fixed points) can be used to analyze its convergence rate. For details, applications, and a generalization to other actions, see [34, 35].

\section{Fixed points on matchings}

Let $M_{2 n}$ be the set of perfect matchings on $2 n$ points. Thus, if $2 n=4, M_{2 n}=$ $\{(1,2)(3,4),(1,3)(2,4),(1,4)(2,3)\}$. It is well known that

$$
\left|M_{2 n}\right|=(2 n-1) ! !=(2 n-1)(2 n-3) \cdots(3)(1) .
$$

The literature on perfect matchings is enormous. See Lovász and Plummer [56] for a book length treatment. Connections with phylogenetic trees and further references are in $[25,26]$. As explained above, the symmetric group $S_{2 n}$ acts primitively on $M_{2 n}$. Results of Luczak and Pyber [57] imply that, in this action, almost every permutation is a derangement. In this section we give sharp asymptotic rates for this last result. We show that the proportion of derangements in $S_{2 n}$ acting on $M_{2 n}$ is

$$
1-\frac{A(1)}{\sqrt{\pi n}}+o\left(\frac{1}{\sqrt{n}}\right), \quad A(1)=\prod_{i=1}^{\infty} \cosh (1 /(2 i-1)) .
$$

Similar asymptotics are given for the proportion of permutations in $S_{2 n}$ acting on $M_{2 n}$ with $j>0$ fixed points. This is zero if $j$ is even. For odd $j$, it is

$$
\frac{C(j) B(1)}{\sqrt{\pi n}}+o\left(\frac{1}{\sqrt{n}}\right), \quad B(1)=\prod_{i=1}^{\infty}\left(1+\frac{1}{2 i}\right) e^{-1 / 2 i}
$$

and $C(j)$ explicit rational numbers. In particular

$$
C(1)=\frac{3}{2}, C(3)=\frac{1}{4}, C(5)=\frac{27}{400}, C(7)=\frac{127}{2352} .
$$


The argument proceeds by finding explicit closed forms for generating functions followed by standard asymptotics. It is well known that the rank of this action is $p(n)$, the number of partitions of $n$. Thus (5.1) is a big improvement over the upper bound given in Theorem 3.1 .

For $w \in S_{2 n}$, let $A_{i}(w)$ be the number of $i$-cycles in the cycle decomposition. Let $F(w)$ be the number of fixed points of $w$ acting on $M_{2 n}$. The following proposition determines $F(w)$ in terms of $A_{i}(w), 1 \leq i \leq 2 n$.

Proposition 5.4 The number of fixed points, $F(w)$ of $w \in S_{2 n}$ on $M_{2 n}$ is

$$
F(w)=\prod_{i=1}^{2 n} F_{i}\left(A_{i}(w)\right)
$$

with

$$
\begin{gathered}
F_{2 i-1}(a)= \begin{cases}1 & \text { if } a=0 \\
0 & \text { if } a \text { is odd } \\
(a-1) ! !(2 i-1)^{a / 2} & \text { if } a>0 \text { is even }\end{cases} \\
F_{2 i}(a)=1+\sum_{k=1}^{\lfloor a / 2\rfloor}(2 k-1) ! !\left(\begin{array}{c}
a \\
2 k
\end{array}\right)(2 i)^{k}
\end{gathered}
$$

In particular,

$$
F(w) \neq 0 \text { if and only if } A_{2 i-1}(w) \text { is even for all } i
$$

$F(w)$ does not take on non-zero even values.

Proof Consider first the cycles of $w$ of length $2 i-1$. If $A_{2 i-1}(w)$ is even, the cycles may be matched in pairs, then each pair of length $2 i-1$ can be broken into matched two element subsets by first pairing the lowest element among the numbers with any of the $2 i-1$ numbers in the second cycle. The rest is determined by cyclic action. For example, if the two three-cycles (123)(456) appear, the matched pairs (14)(25)(36) are fixed, so are (15)(26)(34) or (16)(24)(35). Thus $F_{3}(2)=3$. If $A_{2 i-1}(w)$ is odd, some element cannot be matched and $F_{2 i-1}\left(A_{2 i-1}(w)\right)=0$.

Consider next the cycles of $w$ of length $2 i$. Now, there are two ways to create parts of a fixed perfect matching. First, some of these cycles can be paired and, for each pair, the previous construction can be used. Second, for each unpaired cycle, elements $i$ apart can be paired. For example, from (1234) the pairing (13)(24) may be formed. The sum in $F_{2 i}(a)$ simply enumerates by partial matchings.

To see that $F(w)$ cannot take on non-zero even values, observe that $F_{2 i-1}(a)$ and $F_{2 i}(a)$ only take on odd values if they are non-zero.

Let $P_{2 n}(j)=\frac{\left|\left\{w \in S_{2 n}: F(w)=j\right\}\right|}{2 n !}$. For $j \geq 1$, let $g_{j}(t)=\sum_{n=0}^{\infty} t^{2 n} P_{2 n}(j)$ and let $\bar{g}_{0}(t)=\sum_{n=0}^{\infty} t^{2 n}\left(1-P_{2 n}(0)\right)$. 


\section{Proposition 5.7}

$$
\bar{g}_{0}(t)=\frac{\prod_{i=1}^{\infty} \cosh \left(t^{2 i-1} /(2 i-1)\right)}{\sqrt{1-t^{2}}} \quad \text { for } 0<t<1 \text {. }
$$

Proof From Proposition 5.4, $w \in S_{2 n}$ has $F(w) \neq 0$ if and only if $A_{2 i-1}(w)$ is even for all $i$. From Shepp-Lloyd [66], if $N$ is chosen in $\{0,1,2, \ldots\}$ with

$$
P(N=n)=(1-t) t^{n}
$$

and then $w$ is chosen uniformly in $S_{N}$, the $A_{i}(w)$ are independent Poisson random variables with parameter $t^{i} / i$ respectively. If $X$ is Poisson with parameter $\lambda, P(X$ is even $)=\left(\frac{1}{2}+\frac{e^{-2 \lambda}}{2}\right)$. It follows that

$$
\begin{aligned}
\sum_{n=0}^{\infty}(1-t) t^{2 n}\left(1-P_{2 n}(0)\right) & =\prod_{i=1}^{\infty}\left(\frac{1+e^{-2 t^{2 i-1} /(2 i-1)}}{2}\right) \\
& =\prod_{i=1}^{\infty} e^{-t^{2 i-1} /(2 i-1)} \prod_{i=1}^{\infty} \cosh \left(\frac{t^{2 i-1}}{(2 i-1)}\right) \\
& =\sqrt{\frac{1-t}{1+t}} \prod_{i=1}^{\infty} \cosh \left(t^{2 i-1} /(2 i-1)\right) .
\end{aligned}
$$

Corollary 5.8 As $n$ tends to infinity,

$$
1-P_{2 n}(0) \sim \frac{\prod_{i=1}^{\infty} \cosh (1 /(2 i-1))}{\sqrt{\pi n}} .
$$

Proof By Proposition 5.7, $1-P_{2 n}(0)$ is the coefficient of $t^{n}$ in

$$
\frac{\prod_{i=1}^{\infty} \cosh \left(t^{(2 i-1) / 2} /(2 i-1)\right)}{\sqrt{1-t}} .
$$

It is straightforward to check that the numerator is analytic near $t=1$, so the result follows from Darboux's theorem ([61], Theorem 11.7).

Proposition 5.4 implies that for $j \neq 0$, the event $F(w)=j$ is contained in the event $\left\{A_{2 i-1}(w)\right.$ is even for all $i$ and $A_{2 i}(w) \in\{0,1\}$ for $\left.2 i>j\right\}$. This is evidently complicated for large $j$.

We prove 
Proposition 5.9 For positive odd $j$,

$$
g_{j}(t)=\frac{P_{j}(t) \prod_{i=1}^{\infty}\left(1+\frac{t^{2 i}}{2 i}\right) e^{-t^{2 i} / 2 i}}{\sqrt{1-t^{2}}}
$$

for $P_{j}(t)$ an explicit rational function in $t$ with positive rational coefficients. In particular,

$$
\begin{gathered}
P_{1}(t)=\left(1+\frac{t^{2}}{2}\right), P_{3}(t)=\left(\frac{t^{4}}{6}+\frac{t^{6}}{18}+\frac{t^{8}}{36}\right) \\
P_{5}(t)=\frac{\frac{1}{2}\left(1+\frac{t^{2}}{2}\right)\left(\frac{t^{2}}{4}\right)^{2}}{1+\frac{t^{4}}{4}}+\frac{1}{2}\left(1+\frac{t^{2}}{2}\right)\left(\frac{t^{5}}{5}\right)^{2} \\
P_{7}(t)=\frac{\frac{1}{2}\left(1+\frac{t^{2}}{2}\right)}{1+\frac{t^{6}}{6}}\left(\frac{t^{6}}{6}\right)^{2}+\frac{1}{6}\left(\frac{t^{2}}{2}\right)^{3}+\frac{1}{2}\left(1+\frac{t^{2}}{2}\right)\left(\frac{t^{7}}{7}\right)^{2} .
\end{gathered}
$$

Proof Consider first the case of $j=1$. From Proposition 5.4, $F(w)=1$ if and only if $A_{2 i-1}(w)=0$ for $i \geq 2, A_{1}(w) \in\{0,2\}$ and $A_{2 i}(w) \in\{0,1\}$ for all $i \geq 1$. For example, if $2 n=10$ and $w=(1)(2)(345678910)$, the unique fixed matching is (1 2)(3 7)(4 8)(5 9)(6 10). From the cycle index argument used in Proposition 5.7,

$$
\begin{aligned}
& \sum_{n=0}^{\infty}(1-t) t^{2 n} P_{2 n}(F(w)=1) \\
= & e^{-t}\left(1+\frac{t^{2}}{2}\right) \prod_{i=2}^{\infty} e^{-t^{2 i-1} /(2 i-1)} \prod_{i=1}^{\infty} e^{-t^{2 i} / 2 i}\left(1+\frac{t^{2 i}}{2 i}\right) \\
= & (1-t)\left(1+\frac{t^{2}}{2}\right) \prod_{i=1}^{\infty}\left(1+\frac{t^{2 i}}{2 i}\right) \\
= & \frac{(1-t)\left(1+\frac{t^{2}}{2}\right) \prod_{i=1}^{\infty}\left(1+\frac{t^{2 i}}{2 i}\right) e^{-t^{2 i} / 2 i}}{\sqrt{1-t^{2}}}
\end{aligned}
$$

The arguments for the other parts are similar. In particular, $F(w)=3$ iff one of the following holds:

- $A_{1}(w)=4$, all $A_{2 i-1}(w)=0 i \geq 2$, all $A_{2 i}(w) \in\{0,1\}$

- $A_{1}(w) \in\{0,2\}, A_{2}(w)=2$, all $A_{2 i-1}(w)=0$ and $A_{2 i}(w) \in\{0,1\} i \geq 2$

- $A_{1}(w) \in\{0,2\}, A_{3}(w)=2, A_{2 i-1}(w)=0 i \geq 3, A_{2 i}(w) \in\{0,1\}$

Similarly, $F(w)=5$ iff one of the following holds: 
- $A_{4}(w)=2, A_{1}(w) \in\{0,2\}, A_{2 i-1}(w)=0, A_{2 i}(w) \in\{0,1\}$ else

- $A_{5}(w)=2, A_{1}(w) \in\{0,2\}, A_{2 i-1}(w)=0, A_{2 i}(w) \in\{0,1\}$ else

Finally, $F(w)=7$ iff one of the following holds:

- $A_{1}(w) \in\{0,2\}, A_{6}(w)=2$ or $A_{2}(w)=3, A_{2 i-1}(w)=0, A_{2 i}(w) \in\{0,1\}$ else

- $A_{7}(w)=2, A_{1}(w) \in\{0,2\}, A_{2 i-1}(w)=0, A_{2 i}(w) \in\{0,1\}$ else

Further details are omitted.

The asymptotics in (5.2) follow from Proposition 5.9, by the same method used to prove (5.1) in Corollary 5.8.

\section{More imprimitive subgroups}

Section 5 studied fixed points on matchings, or equivalently fixed points of $S_{2 n}$ on the left cosets of $S_{2}$ ₹ $S_{n}$. This section uses a quite different approach to study derangements of $S_{a n}$ on the left cosets of $S_{a} 2 S_{n}$, where $a \geq 2$ is constant. It is proved that the proportion of elements of $S_{a n}$ which fix at least one left coset of $S_{a}$ 2 $S_{n}$ (or equivalently are conjugate to an element of $S_{a}$ 2 $S_{n}$ or equivalently fix a system of $n$ blocks of size $a$ ) is at most the coefficient of $u^{n}$ in

$$
\exp \left(\sum_{k \geq 1} \frac{u^{k}}{a !}\left(\frac{1}{k}\right)\left(\frac{1}{k}+1\right) \cdots\left(\frac{1}{k}+a-1\right)\right),
$$

and that this coefficient is asymptotic to $C_{a} n^{\frac{1}{a}-1}$ as $n \rightarrow \infty$, where $C_{a}$ is an explicit constant depending on $a$ (defined in Theorem 6.3 below). In the special case of matchings $(a=2)$, this becomes $\frac{e^{\pi^{2} / 12}}{\sqrt{\pi n}}$, which is extremely close to the true asymptotics obtained in Section 5. Moreover, this generating function will be crucially applied when we sharpen a result of Luczak and Pyber in Section 7.

The method of proof is straightforward. Clearly the number of permutations in $S_{a n}$ conjugate to an element of $S_{a} 2 S_{n}$ is upper bounded by the sum over conjugacy classes $C$ of $S_{a}$ ₹ $S_{n}$ of the size of the $S_{a n}$ conjugacy class of $C$. Unfortunately this upper bound is hard to compute, but we show it to be smaller than something which can be exactly computed as a coefficient in a generating function. This will prove the result.

From Section 4.2 of [47], there is the following useful description of conjugacy classes of $G$ ₹ $S_{n}$ where $G$ is a finite group. The classes correspond to matrices $M$ with natural number entries $M_{i, k}$, rows indexed by the conjugacy classes of $G$, columns indexed by the numbers $1,2, \cdots, n$, and satisfying the condition that $\sum_{i, k} k M_{i, k}=n$. More precisely, given an element $\left(g_{1}, \cdots, g_{n} ; \pi\right)$ in $G<S_{n}$, for each k-cycle of $\pi$ one multiplies the $g$ 's whose subscripts are the elements of the cycle in the order specified by the cycle. Taking the conjugacy class in $G$ of the resulting product contributes 1 to the matrix entry whose row corresponds to this conjugacy class in $G$ and whose column is $k$. 
The remainder of this section specializes to $G=S_{a}$. Since conjugacy classes of $S_{a}$ correspond to partitions $\lambda$ of $a$, the matrix entries are denoted by $M_{\lambda, k}$. We write $|\lambda|=a$ if $\lambda$ is a partition of $a$. Given a partition $\lambda$, let $n_{i}(\lambda)$ denote the number of parts of size $i$ of $\lambda$.

Proposition 6.1 Let the conjugacy class $C$ of $S_{a} 2 S_{n}$ correspond to the matrix $\left(M_{\lambda, k}\right)$ where $\lambda$ is a partition of $a$. Then the proportion of elements of $S_{a n}$ conjugate to an element of $C$ is at most

$$
\frac{1}{\prod_{k} \prod_{|\lambda|=a} M_{\lambda, k} !\left[\prod_{i}(i k)^{n_{i}(\lambda)} n_{i}(\lambda) !\right]^{M_{\lambda, k}}} .
$$

Proof Observe that the number of cycles of length $j$ of an element of $C$ is equal to

$$
\sum_{k \mid j} \sum_{|\lambda|=a} M_{\lambda, k} n_{j / k}(\lambda)
$$

To see this, note that $S_{a}$ 2 $S_{n}$ can be viewed concretely as a permutation of an symbols by letting it act on an array of $n$ rows of length $a$, with $S_{a}$ permuting within each row and $S_{n}$ permuting among the rows.

Hence by a well known formula for conjugacy class sizes in a symmetric group, the proportion of elements of $S_{a n}$ conjugate to an element of $C$ is equal to

$$
\begin{aligned}
& \frac{1}{\prod_{j} j^{\sum_{k \mid j} \sum_{|\lambda|=a} M_{\lambda, k} n_{j / k}(\lambda)}\left[\sum_{k \mid j} \sum_{|\lambda|=a} M_{\lambda, k} n_{j / k}(\lambda)\right] !} \\
\leq & \frac{1}{\prod_{j} j^{\sum_{k \mid j} \sum_{|\lambda|=a} M_{\lambda, k} n_{j / k}(\lambda)} \prod_{k \mid j} \prod_{|\lambda|=a} M_{\lambda, k} n_{j / k}(\lambda) !} \\
\leq & \frac{1}{\prod_{j} j^{\sum_{k \mid j} \sum_{|\lambda|=a} M_{\lambda, k} n_{j / k}(\lambda)} \prod_{k \mid j} \prod_{|\lambda|=a}\left[M_{\lambda, k} ! n_{j / k}(\lambda) ! M_{\lambda, k}\right]} \\
= & \frac{1}{\prod_{k} \prod_{|\lambda|=a} M_{\lambda, k} !\left[\prod_{i}(i k)^{n_{i}(\lambda)} n_{i}(\lambda) !\right]^{M_{\lambda, k}}},
\end{aligned}
$$

as desired. The first inequality uses the fact that $\left(x_{1}+\cdots+x_{n}\right) ! \geq x_{1} ! \cdots x_{n}$ !. The second inequality uses that $(x y) ! \geq x ! y !^{x}$ for $x, y \geq 1$ integers, which is true since

$$
(x y) !=\prod_{i=1}^{x} \prod_{j=0}^{y-1}(i+j x) \geq \prod_{i=1}^{x} \prod_{j=0}^{y-1} i(1+j)=(x !)^{y}(y !)^{x} \geq x !(y !)^{x} .
$$

The final equality used the change of variables $i=j / k$.

To proceed further, the next lemma is useful. 


\section{Lemma 6.2}

$$
\sum_{|\lambda|=a} \frac{1}{\prod_{i}(i k)^{n_{i}(\lambda)} n_{i}(\lambda) !}=\frac{\left(\frac{1}{k}\right)\left(\frac{1}{k}+1\right) \cdots\left(\frac{1}{k}+a-1\right)}{a !} .
$$

Proof Let $c(\pi)$ denote the number of cycles of a permutation $\pi$. Since the number of permutations in $S_{a}$ with $n_{i}$ cycles of length $i$ for each $i$ is $\frac{a !}{\prod_{i} i^{n_{i}} n_{i} !}$, the left hand side is equal to

$$
\frac{1}{a !} \sum_{\pi \in S_{a}} k^{-c(\pi)}
$$

It is well known and easily proved by induction that

$$
\sum_{\pi \in S_{a}} x^{c(\pi)}=x(x+1) \cdots(x+a-1) .
$$

Theorem 6.3 applies the preceding results to obtain a useful generating function.

\section{Theorem 6.3}

(1) The proportion of elements in $S_{a n}$ conjugate to an element of $S_{a} 2 S_{n}$ is at most the coefficient of $u^{n}$ in

$$
\exp \left(\sum_{k \geq 1} \frac{u^{k}}{a !}\left(\frac{1}{k}\right)\left(\frac{1}{k}+1\right) \cdots\left(\frac{1}{k}+a-1\right)\right) .
$$

(2) For a fixed and $n \rightarrow \infty$, the coefficient of $u^{n}$ in this generating function is asymptotic to

$$
\frac{e^{\sum_{r=2}^{a} p(a, r) \zeta(r)}}{\Gamma(1 / a)} n^{\frac{1}{a}-1}
$$

where $p(a, r)$ is the proportion of permutations in $S_{a}$ with exactly $r$ cycles, $\zeta$ is the Riemann zeta function, and $\Gamma$ is the gamma function.

Proof Proposition 6.1 implies that the sought proportion is at most the coefficient of $u^{n}$ in

$$
\begin{aligned}
& \prod_{k} \prod_{|\lambda|=a} \sum_{M_{\lambda, k} \geq 0} \frac{u^{k M_{\lambda, k}}}{M_{\lambda, k} !\left[(i k)^{n_{i}(\lambda)} n_{i}(\lambda) !\right]^{M_{\lambda, k}}} \\
= & \prod_{k} \prod_{|\lambda|=a} \exp \left(\frac{u^{k}}{(i k)^{n_{i}(\lambda)} n_{i}(\lambda) !}\right) \\
= & \prod_{k} \exp \left(\sum_{|\lambda|=a} \frac{u^{k}}{(i k)^{n_{i}(\lambda)} n_{i}(\lambda) !}\right)
\end{aligned}
$$




$$
=\exp \left(\sum_{k \geq 1} \frac{u^{k}}{a !}\left(\frac{1}{k}\right)\left(\frac{1}{k}+1\right) \cdots\left(\frac{1}{k}+a-1\right)\right) .
$$

The last equality used Lemma 6.2.

For the second assertion, one uses Darboux's lemma (see [61] for an exposition), which gives the asymptotics of functions of the form $(1-u)^{\alpha} g(u)$ where $g(u)$ is analytic near $1, g(1) \neq 0$, and $\alpha \notin\{0,1,2, \cdots\}$. More precisely it gives that the coefficient of $u^{n}$ in $(1-u)^{\alpha} g(u)$ is asymptotic to $\frac{g(1)}{\Gamma(-\alpha)} n^{-\alpha-1}$. By Lemma 6.2,

$$
\begin{aligned}
& \exp \left(\sum_{k \geq 1} \frac{u^{k}}{a !}\left(\frac{1}{k}\right)\left(\frac{1}{k}+1\right) \cdots\left(\frac{1}{k}+a-1\right)\right) \\
= & \exp \left(\sum_{k \geq 1} \frac{u^{k}}{a k}+\sum_{k \geq 1} u^{k} \sum_{r=2}^{a} p(a, r) k^{-r}\right) \\
= & (1-u)^{-\frac{1}{a}} \cdot \exp \left(\sum_{r=2}^{a} p(a, r) \sum_{k \geq 1} \frac{u^{k}}{k^{r}}\right) .
\end{aligned}
$$

Taking $g(u)=\exp \left(\sum_{r=2}^{a} p(a, r) \sum_{k \geq 1} \frac{u^{k}}{k^{r}}\right)$ proves the result.

Remark The upper bound in Theorem 6.3 is not perfect. In fact when $n=2$, it does not approach 0 as $a \rightarrow \infty$, whereas the true answer must by Theorem 3.4. However by part 2 of Theorem 6.3 , the bound is useful for $a$ fixed and $n$ growing, and it will be crucially applied in Section 7 when $a=n$ are both growing.

\section{Primitive subgroups}

A main goal of this section is to prove that the proportion of elements of $S_{n}$ which belong to a primitive subgroup not containing $A_{n}$ is at most $O\left(n^{-2 / 3+\alpha}\right)$ for any $\alpha>0$. This improves on the bound $O\left(n^{-1 / 2+\alpha}\right)$ in [57], which was used in proving Theorem 3.3 in Section 2. We conjecture that this can in fact be replaced by $O\left(n^{-1}\right)$ (and the examples with $n=\left(q^{d}-1\right) /(q-1)$ with the subgroup containing $P G L(d, q)$ or $n=p^{d}$ with subgroup $A G L(d, p)$ show that in general one can do no better).

The minimal degree of a permutation group is defined as the least number of points moved by a nontrivial element. The first step is to classify the degree $n$ primitive permutation groups with minimal degree at most $n^{2 / 3}$. We note that Babai [6] gave an elegant proof (not requiring the classification of finite simple groups) that there are no primitive permutation groups of degree $n$ other than $A_{n}$ or $S_{n}$ with minimal degree at most $n^{1 / 2}$.

Theorem 7.1 Let $G$ be a primitive permutation group of degree $n$. Assume that there is a nontrivial $g \in G$ moving fewer than $n^{2 / 3}$ points. Then one of the following holds: 
(1) $G=A_{n}$ or $S_{n}$;

(2) $G=S_{m}$ or $A_{m}$ with $m \geq 5$ and $n=\left(\begin{array}{c}m \\ 2\end{array}\right)$ (acting on subsets of size 2); or

(3) $A_{m} \times A_{m}<G \leq S_{m} 2 S_{2}$ with $m \geq 4$ and $n=m^{2}$ (preserving a product structure).

If there is a nontrivial $g \in G$ moving fewer than $n^{1 / 2}$ points, then $G=A_{n}$ or $S_{n}$.

Proof First note that the minimal degree in $(2)$ is $2(m-2)$ and in (3) is $2 n^{1 / 2}$. In particular, aside from (1), we always have the minimal degree is at least $n^{1 / 2}$. Thus, the last statement follows from the first part.

It follows by the main result of [45] that if there is a $g \in G$ moving fewer than $n / 2$ points, then one the following holds:

(a) $G$ is almost simple with socle (the subgroup generated by the minimal normal subgroups) $A_{m}$ and $n=\left(\begin{array}{c}m \\ k\end{array}\right)$ with the action on subsets of size $k<m / 2$;

(b) $n=m^{t}$, with $t>1, m \geq 5, G$ has a unique minimal normal subgroup $N=$ $L_{1} \times \ldots \times L_{t}$ with $t>1$ and $L_{i} \cong L$, a non-Abelian simple group; moreover $G$ preserves a product structure - i.e. if $\Omega=\{1, \ldots, n\}$, then as $G$-sets, $\Omega \cong X^{t}$ where $m=|X|, G \leq S_{m}$ 2 $S_{t}$ acts on $X^{t}$ by acting on each coordinate and permuting the coordinates.

Note that $n / 2 \geq n^{2 / 3}$ as long as $n \geq 8$. If $n<8$, then $G$ contains an element moving at most 3 points, i.e. either a transposition or a 3 cycle, and so contains $A_{n}$ (Theorem 3.3A in [31]).

Consider (a) above. If $k=1$, then (1) holds. If $3 \leq k<m / 2$, then it is an easy exercise to see that the element of $S_{m}$ moving the fewest $k$-sets is a transposition. The number of $k$-sets moved is $2\left(\begin{array}{c}m-2 \\ k-1\end{array}\right)$. We claim that this is greater than $n^{2 / 3}$. Indeed, the sought inequality is equivalent to checking that $\frac{2 k(m-k)}{m(m-1)}>\left(\begin{array}{c}m \\ k\end{array}\right)^{-1 / 3}$. The worst case is clearly $k=3$, which is checked by taking cubes. This settles the case $3 \leq k<m / 2$, and if $k=2$, we are in case (2).

Now consider (b) above. Suppose that $t \geq 3$. Then if $g \in S_{m} \times \cdots \times S_{m}$ is nontrivial, it moves at least $2 m^{t-1}>n^{2 / 3}$ many points. If $g \in S_{m} 2 S_{t}$ and is not in $S_{m} \times \cdots \times S_{m}$, then up to conjugacy we may write $g=\left(g_{1}, \cdots, g_{t} ; \sigma\right)$ where say $\sigma$ has an orbit $\{1, \ldots, s\}$ with $s>1$. Viewing our set as $A \times B$ with $A$ being the first $s$ coordinates, we see that $g$ fixes at most $m$ points on $A$ (since there is at most one $g$ fixed point with a given coordinate) and so on the whole space, $g$ fixes at most $m^{t-s+1} \leq m^{t-1}$ points and so moves at least $m^{t}-m^{t-1}$ points. Since $t \geq 3$, this is greater than $n^{2 / 3}$. Summarizing, we have shown that in case (b), $t \geq 3$ leads to a contradiction.

So finally consider (b) with $t=2$. We claim that $L$ must be $A_{m}$. Enlarging the group slightly, we may assume that $G=S 2 S_{2}$ where $L \leq S \leq A u t(L)$ and $S$ is primitive of degree $m$. If $g \notin S \times S$, then arguing as in the $t=3$ case shows that $g$ moves at least $m^{2}-m$ points. This is greater than $m^{4 / 3}=n^{2 / 3}$ since $m \geq 5$, a contradiction. So write $g=\left(g_{1}, g_{2}\right) \in S \times S$ with say $g_{1} \neq 1$. If $g_{1}$ moves at least $d$ points, then $g$ moves at least $d m$ points. This is greater than $n^{2 / 3}$ unless $d \leq m^{1 / 3}$. Arguing inductively (with $n$ replaced by $m$ in the theorem we are proving), this implies that $L=A_{m}$, whence (1) holds.

Next, we focus on Case 2 of Theorem 7.1. 
Lemma 7.2 Let $S_{m}$ be viewed as a subgroup of $S_{\left(\begin{array}{c}m \\ 2\end{array}\right)}$ using its action on 2-sets of $\{1, \cdots, m\}$. For $w \in S_{m}$, let $A_{i}(w)$ denote the number of cycles of $w$ of length $i$ in its usual action on $\{1, \cdots, m\}$. The total number of orbits of $w$ on 2-sets $\{j, k\}$ of symbols which are in a common cycle of $w$ is

$$
\frac{m}{2}-\sum_{i \text { odd }} \frac{A_{i}(w)}{2}
$$

Proof First suppose that $w$ is a single cycle of length $i \geq 2$. If $i$ is odd, then all orbits of $w$ on pairs of symbols in the $i$-cycle have length $i$, so the total number of orbits is $\frac{\left(\begin{array}{c}i \\ 2\end{array}\right)}{i}=\frac{i-1}{2}$. If $i$ is even, there is 1 orbit of size $\frac{i}{2}$ and all other orbits have size $i$, giving a total of $\frac{i}{2}$ orbits. Hence for general $w$, the total number of orbits on pairs of symbols in a common cycle of $w$ is

$$
\begin{aligned}
\sum_{\substack{i \text { odd } \\
i \geq 3}} A_{i}(w) \frac{i-1}{2}+\sum_{i \text { even }} A_{i}(w) \frac{i}{2} & =\sum_{\substack{i \text { odd } \\
i \geq 1}} A_{i}(w) \frac{i-1}{2}+\sum_{i \text { even }} A_{i}(w) \frac{i}{2} \\
& =\frac{m}{2}-\sum_{i \text { odd }} \frac{A_{i}(w)}{2} .
\end{aligned}
$$

Theorem 7.3 Let $S_{m}$ be viewed as a subgroup of $S_{n}$ with $n=\left(\begin{array}{c}m \\ 2\end{array}\right)$ using its action on 2-sets of $\{1, \cdots, m\}$. Then the proportion of elements of $S_{n}$ contained in a conjugate of $S_{m}$ is at most $O\left(\frac{\log (n)}{n}\right)$.

Proof We claim that any element $w$ of $S_{m}$ has at least $\frac{m}{12}$ cycles when viewed as an element of $S_{n}$. Indeed, if $A_{1}(w)>\frac{m}{2}$, then $w$ fixes at least $\frac{m(m-1)}{8} \geq \frac{m}{12}$ two-sets. So we suppose that $A_{1}(w) \leq \frac{m}{2}$. Clearly $\sum_{i \geq 3 \text { odd }} A_{i}(w) \leq \frac{m}{3}$. Thus Lemma 7.2 implies that $w$ has at least $\frac{m}{2}-\frac{m}{4}-\frac{m}{6}=\frac{m}{12}$ cycles as an element of $S_{n}$. The number of cycles of a random element of $S_{n}$ has mean and variance asymptotic to $\log (n) \sim$ $2 \log (m)$ (and is in fact asymptotically normal) [41]. Thus by Chebyshev's inequality, the proportion of elements in $S_{m}$ with at least $\frac{m}{12}$ cycles is $O\left(\frac{\log (m)}{m^{2}}\right)=O\left(\frac{\log (n)}{n}\right)$, as desired.

To analyze Case 3 of Theorem 7.1, the following bound, based on the generating function from Section 6, will be needed.

Proposition 7.4 The proportion of elements in $S_{m^{2}}$ which fix a system of $m$ blocks of size $m$ is $O\left(n^{-3 / 4+\alpha}\right)$ for any $\alpha>0$.

Proof By Theorem 6.3, the proportion in question is at most the coefficient of $u^{m}$ in

$$
\exp \left(\sum_{k \geq 1} \frac{u^{k}}{m k}\left(1+\frac{1}{k}\right)\left(1+\frac{1}{2 k}\right) \cdots\left(1+\frac{1}{(m-1) k}\right)\right) \text {. }
$$


If $f(u)$ and $g(u)$ are power series in $u$, we write $f(u)<<g(u)$ if the coefficient of $u^{n}$ in $f(u)$ is less than or equal to the corresponding coefficient in $g(u)$, for all $n$. Since $\log (1+x) \leq x$ for $0<x<1$, one has that

$$
\log \left(\prod_{i=1}^{m-1}\left(1+\frac{1}{i k}\right)\right) \leq \sum_{i=1}^{m-1} \frac{1}{k i} \leq \frac{1}{k}(1+\log (m-1)) .
$$

Thus

$$
\begin{aligned}
& \exp \left(\sum_{k \geq 1} \frac{u^{k}}{m k}\left(1+\frac{1}{k}\right)\left(1+\frac{1}{2 k}\right) \cdots\left(1+\frac{1}{(m-1) k}\right)\right) \\
< & \exp \left(\sum_{k \geq 1} \frac{u^{k}}{m k} e^{1 / k}(m-1)^{1 / k}\right) \\
<< & e^{u e} \exp \left(\sum_{k \geq 2} \frac{u^{k}}{k} \sqrt{\frac{e}{m}}\right) \\
<< & e^{u e} \exp \left(\sum_{k \geq 1} \frac{u^{k}}{k} \sqrt{\frac{e}{m}}\right) \\
= & e^{u e}(1-u)^{-\sqrt{\frac{e}{m}}} .
\end{aligned}
$$

The coefficient of $u^{i}$ in $(1-u)^{-\sqrt{\frac{e}{m}}}$ is

$$
\frac{1}{i !} \sqrt{\frac{e}{m}} \prod_{j=1}^{i-1}\left(\sqrt{\frac{e}{m}}+j-1\right)=\frac{1}{i} \sqrt{\frac{e}{m}} \prod_{j=1}^{i-1}\left(1+\frac{1}{j} \sqrt{\frac{e}{m}}\right) .
$$

Since

$$
\log \left(\prod_{j=1}^{i-1}\left(1+\frac{1}{j} \sqrt{\frac{e}{m}}\right)\right) \leq \sum_{j=1}^{i-1} \frac{1}{j} \sqrt{\frac{e}{m}} \leq \sqrt{\frac{e}{m}}(1+\log (i-1)),
$$

it follows that

$$
\prod_{j=1}^{i-1}\left(1+\frac{1}{j} \sqrt{\frac{e}{m}}\right) \leq[e(i-1)]^{\sqrt{\frac{e}{m}}} .
$$

This is at most a universal constant $A$ if $0 \leq i \leq m$. Thus the coefficient of $u^{m}$ in $e^{u e}(1-u)^{-\sqrt{\frac{e}{m}}}$ is at most

$$
\frac{e^{m}}{m !}+A \sqrt{\frac{e}{m}} \sum_{i=1}^{m} \frac{1}{i} \frac{e^{m-i}}{(m-i) !}
$$


By Stirling's formula [33], $m !>m^{m} e^{-m+1 /(12 m+1)} \sqrt{2 \pi m}$, which implies that the first term is very small for large $m$. To bound the sum, consider the terms for $i \geq m^{1-\alpha}$, where $0<\alpha<1$. These contribute at most $\frac{B m^{\alpha}}{m^{3 / 2}}$ for a universal constant $B$. The contribution of the other terms is negligible in comparison, by Stirling's formula. Summarizing, the contribution of the sum is $O\left(m^{-3 / 2+\alpha}\right)=O\left(n^{-3 / 4+\alpha / 2}\right)$, as desired.

The following theorem gives a bound for Case 3 of Theorem 7.1.

Theorem 7.5 Let $S_{m} \geq S_{2}$ be viewed as a subgroup of $S_{n}$ with $n=m^{2}$ using its action on the Cartesian product $\{1, \cdots, m\}^{2}$. Then the proportion of elements of $S_{n}$ conjugate to an element of $S_{m} 2 S_{2}$ is $O\left(n^{-3 / 4+\alpha}\right)$ for any $\alpha>0$.

Proof Consider elements of $S_{m} \imath S_{2}$ of the form $\left(w_{1}, w_{2} ; i d\right)$. These all fix $m$ blocks of size $m$ in the action on $\{1, \cdots, m\}^{2}$; the blocks consist of points with a given first coordinate. By Proposition 7.4, the proportion of elements of $S_{n}$ conjugate to some $\left(w_{1}, w_{2} ; i d\right)$ is $O\left(n^{-3 / 4+\alpha}\right)$ for any $\alpha>0$.

Next, consider an element of $S_{m} 2 S_{2}$ of the form $\sigma=\left(w_{1}, w_{2} ;(12)\right)$. Then $\sigma^{2}=$ $\left(w_{1} w_{2}, w_{2} w_{1} ; i d\right)$. Note that $w_{1} w_{2}$ and $w_{2} w_{1}$ are conjugate in $S_{m}$, and let $A_{i}$ denote their common number of $i$-cycles. Observe that if $x$ is in an $i$-cycle of $w_{1} w_{2}$, and $y$ is in an $i$-cycle of $w_{2} w_{1}$, then $(x, y) \in \Omega$ is in an orbit of $\sigma^{2}$ of size $i$. Hence the total number of orbits of $\sigma^{2}$ of size $i$ is at least $\frac{\left(i A_{i}\right)^{2}}{i} \geq i A_{i}$. Thus the total number of orbits of $\sigma^{2}$ on $\Omega$ is at least $\sum_{i} i A_{i}=m$. Hence the total number of orbits of $\sigma$ is at least $\frac{m}{2}$. Arguing as in the proof of Theorem 7.3, it follows that the proportion of elements of $S_{n}$ conjugate to an element of the form $\sigma$ is $O\left(\frac{\log (n)}{n}\right)$, and so is $O\left(n^{-3 / 4+\alpha}\right)$ for any $\alpha>0$.

Now the main result of this section can be proved.

Theorem 7.6 The proportion of elements of $S_{n}$ which belong to a primitive subgroup not containing $A_{n}$ is at most $O\left(n^{-2 / 3+\alpha}\right)$ for any $\alpha>0$.

Proof Fix $\alpha>0$. By Bovey [13], the proportion of elements $w$ of $S_{n}$ such that $\langle w\rangle$ has minimum degree greater than $n^{2 / 3}$ is $O\left(n^{-2 / 3+\alpha}\right)$. Thus the proportion of $w \in S_{n}$ which lie in a primitive permutation group having minimal degree greater than $n^{2 / 3}$ is $O\left(n^{-2 / 3+\alpha}\right)$. The only primitive permutation groups of degree $n$ with minimal degree $\leq n^{2 / 3}$, and not containing $A_{n}$ are given by Cases 2 and 3 of Theorem 7.1. Theorems 7.3 and 7.5 imply that the proportion of $w$ lying in the union of all such subgroups is $O\left(n^{-2 / 3+\alpha}\right)$, so the result follows.

A trivial corollary to the theorem is that this holds for $A_{n}$ as well.

Remark The actions of the symmetric group studied in this section embed the group as a subgroup of various larger symmetric groups. Any such embedding can be thought of as a code in the larger symmetric group. Such codes may be used for 
approximating sums of various functions over the larger symmetric group via a sum over the smaller symmetric group. Our results can be interpreted as giving examples of functions where the approximation is not particularly accurate. For example, the proof of Theorem 7.3 shows this to be the case when $S_{m}$ is viewed as a subgroup of $S_{n}, n=\left(\begin{array}{c}m \\ 2\end{array}\right)$ using the actions on 2-sets, and the function is the number of cycles.

\section{Related results and applications}

There are numerous applications of the distribution of fixed points and derangements. Subsection 8.1 mentions some motivation from number theory. Subsection 8.2 discusses some literature on the proportion of derangements and an analog of the main result of our paper for finite classical groups. Subsection 8.3 discusses fixed point ratios, emphasizing the application to random generation. Subsection 8.4 collects some miscellany about fixed points and derangements, including algorithmic issues and appearances in algebraic combinatorics.

While this section does cover many topics, the survey is by no means comprehensive. Some splendid presentations of several other topics related to derangements are Serre [63], Cameron's lecture notes [16] and Section 6.6 of [15]. For the connections with permutations with restricted positions and rook polynomials see [67, 2.3, 2.4].

\subsection{Motivation from number theory}

We describe two number theoretic applications of derangements which can be regarded as motivation for their study:

(1) Zeros of polynomials Let $h(T)$ be a polynomial with integer coefficients which is irreducible over the integers. Let $\pi(x)$ be the number of primes $\leq x$ and let $\pi_{h}(x)$ be the number of primes $\leq x$ for which $h$ has no zeros mod $p$. It follows from Chebotarev's density theorem (see [52] for history and a proof sketch), that $\lim _{x \rightarrow \infty} \frac{\pi_{h}(x)}{\pi(x)}$ is equal to the proportion of derangements in the Galois group $G$ of $h(T)$ (viewed as permutation of the roots of $h(T)$ ). Several detailed examples are worked out in Serre's survey [63].

In addition, there are applications such as the number field sieve for factoring integers (Section 9 of [14]), where it is important to understand the proportion of primes for which $h$ has no zeros mod $p$. This motivated Lenstra (1990) to pose the question of finding a good lower bound for the proportion of derangements of a transitive permutation group acting on a set of $n$ letters with $n \geq 2$. Results on this question are described in Subsection 8.2.

(2) The value problem Let $\mathbb{F}_{q}$ be a finite field of size $q$ with characteristic $p$ and let $f(T)$ be a polynomial of degree $n>1$ in $\mathbb{F}_{q}[T]$ which is not a polynomial in $T^{p}$. The arithmetic question raised by Chowla [19] is to estimate the number $V_{f}$ of distinct values taken by $f(T)$ as $T$ runs over $\mathbb{F}_{q}$.

There is an asymptotic formula for $V_{f}$ in terms of certain Galois groups and derangements. More precisely, let $G$ be the Galois group of $f(T)-t=0$ over $\mathbb{F}_{q}(t)$ 
and let $N$ be the Galois group of $f(T)-t=0$ over $\overline{\mathbb{F}}_{q}(t)$, where $\overline{\mathbb{F}}_{q}$ is an algebraic closure of $\mathbb{F}_{q}$ (we are viewing $f(T)-t$ as a polynomial with variable $T$ with coefficients in $\left.\mathbb{F}_{q}(t)\right)$. Both groups act transitively on the $n$ roots of $f(T)-t=0$. The geometric monodromy group $N$ is a normal subgroup of the arithmetic monodromy group $G$. The quotient group $G / N$ is a cyclic group (possibly trivial).

Theorem 8.1 ([20]) Let $x N$ be the coset which is the Frobenius generator of the cyclic group $G / N$. The Chebotarev density theorem for function fields yields the following asymptotic formula:

$$
V_{f}=\left(1-\frac{\left|S_{0}\right|}{|N|}\right) q+O(\sqrt{q})
$$

where $S_{0}$ is the set of group elements in the coset $x N$ which act as derangements on the set of roots of $f(T)-t=0$. The constant in the above error term depends only on $n$, not on $q$.

As an example, let $f(T)=T^{r}$ with $r$ prime and different from $p$ (the characteristic of the base field $\left.\mathbb{F}_{q}\right)$. The Galois closure of $\mathbb{F}_{q}(T) / \mathbb{F}_{q}\left(T^{r}\right)$ is $\mathbb{F}_{q}(\mu, T)$ where $\mu$ is a nontrivial $r$ th root of 1 . Thus $N$ is cyclic of order $r$ and $G / N$ is isomorphic to the Galois group of $\mathbb{F}_{q}(\mu) / \mathbb{F}_{q}$. The permutation action is of degree $r$. If $G=N$, then every non-trivial element is a derangement and so the image of $f$ has order roughly $\frac{q}{r}+O(\sqrt{q})$. If $G \neq N$, then $G$ is a Frobenius group and every fixed point free element is contained in $N$. Indeed, since in this case $(r, q-1)=1$, we see that $T^{r}$ is bijective on $\mathbb{F}_{q}$. For further examples, see Guralnick-Wan [46] and references therein. Using work on derangements, they prove that if the degree of $f$ is relatively prime to the characteristic $p$, then either $f$ is bijective or $V_{f} \leq \frac{5 q}{6}+O(\sqrt{q})$.

\subsection{Proportion of derangements and Shalev's conjecture}

Let $G$ be a finite permutation group acting transitively on a set $X$ of size $n>1$. Subsection 8.1 motivated the study of $\delta(G, X)$, the proportion of derangements of $G$ acting on $X$. We describe some results on this question, focusing particularly on lower bounds and analogs of our main results for classical groups.

Perhaps the earliest such result is due to Jordan [48], who showed that $\delta(G, X)>$ 0 . Cameron and Cohen [17] proved that $\delta(G, X) \geq 1 / n$ with equality if and only if $G$ is a Frobenius group of order $n(n-1)$, where $n$ is a prime power ([3, p. 193] gives background on Frobenius groups). For further discussion, including a topological application of Jordan's theorem, see [63].

Based on extensive computations, it was asked in [12] whether there is a universal constant $\delta>0$ (which they speculate may be optimally chosen as $\frac{2}{7}$ ) such that $\delta(G, X)>\delta$ for all finite simple groups $G$. The existence of such a $\delta>0$ was also conjectured by Shalev.

Shalev's conjecture was proved by Fulman and Guralnick in the series of papers [36-38]. We do not attempt to sketch a proof of Shalev's conjecture here, but make a few remarks: 
(1) One can assume that the action of $G$ on $X$ is primitive, for if $f: Y \mapsto X$ is a surjection of $G$-sets, then $\delta(G, Y) \geq \delta(G, X)$.

(2) By Jordan's theorem [48] that $\delta(G, X)>0$, the proof of Shalev's conjecture is an asymptotic result: we only need to show that there exists a $\delta>0$ such that for any sequence $G_{i}, X_{i}$ with $\left|X_{i}\right| \rightarrow \infty$, one has that $\delta\left(G_{i}, X_{i}\right)>\delta$ for all sufficiently large $i$.

(3) When $G$ is the alternating group, by Theorem 3.4, for all primitive actions of $A_{n}$ except the action on $k$-sets, the proportion of derangements tends to 1 . For the case of $A_{n}$ on $k$-sets, one can give arguments similar to those Dixon [29], who proved that the proportion of elements of $S_{n}$ which are derangements on $k$-sets is at least $\frac{1}{3}$.

(4) When $G$ is a finite Chevalley group, the key is to study the set of regular semisimple elements of $G$. Typically (there are some exceptions in the orthogonal cases) this is the set of elements of $G$ whose characteristic polynomial is square-free. Now a regular semisimple element is contained in a unique maximal torus, and there is a map from maximal tori to conjugacy classes of the Weyl group. This allows one to relate derangements in $G$ to derangements in the Weyl group. For example, one concludes that the proportion of elements of $G L(n, q)$ which are regular semisimple and fix some $k$-space is at most the proportion of elements in $S_{n}$ which fix a $k$-set. For large $q$, algebraic group arguments show that nearly all elements of $G L(n, q)$ are regular semisimple, and for fixed $q$, one uses generating functions to uniformly bound the proportion of regular semisimple elements away from 0 .

To close this subsection, we note that the main result of this paper has an analog for finite classical groups. The following result was stated in [36] and is proved in [37].

Theorem 8.2 Let $G_{i}$ be a sequence of classical groups with the natural module of dimension $d_{i}$. Let $X_{i}$ be a $G_{i}$-orbit of either totally singular or nondegenerate subspaces (of the natural module) of dimension $k_{i} \leq d_{i} / 2$. If $k_{i} \rightarrow \infty$, then $\lim \delta\left(G_{i}, X_{i}\right)=1$. If $k_{i}$ is a bounded sequence, then there exist $0<\delta_{1}<\delta_{2}<1$ so that $\delta_{1}<\delta\left(G_{i}, X_{i}\right)<\delta_{2}$.

This result applies to any subgroup between the classical group and its socle. Note that in the case that $G_{i}=P S L$, we view all subspaces as being totally singular (note that the totally singular spaces have parabolic subgroups as stabilizers). We also remark that in characteristic 2 , we consider the orthogonal group inside the symplectic group as the stabilizer of a subspace (indeed, if we view $\operatorname{Sp}\left(2 m, 2^{e}\right)=O\left(2 m+1,2^{e}\right)$, then the orthogonal groups are stabilizers of nondegenerate hyperplanes).

In fact, Fulman and Guralnick prove an analog of the Luczak-Pyber result for symmetric groups. This result was proved by Shalev [64] for $P G L(d, q)$ with $q$ fixed.

Theorem 8.3 Let $G_{i}$ be a sequence of simple classical groups with the natural module $V_{i}$ of dimension $d_{i}$ with $d_{i} \rightarrow \infty$. Let $H_{i}$ be the union of all proper irreducible subgroups (excluding orthogonal subgroups of the symplectic group in characteristic 2). Then $\lim _{i \rightarrow \infty}\left|H_{i}\right| /\left|G_{i}\right|=0$. 
If the $d_{i}$ are fixed, then this result is false. For example, if $G_{i}=P S L(2, q)$ and $H$ is the normalizer of a maximal torus of $G$, then $\lim _{q \rightarrow \infty} \delta(G, G / H)=1 / 2$. However, the analog of the previous theorem is proved in [36] if the rank of the Chevalley group is fixed. In this case, we take $H_{i}$ to be the union of maximal subgroups which do not contain a maximal torus.

The example given above shows that the rank of the permutation action going to $\infty$ does not imply that the proportion of derangements tends to 1 . The results of Fulman and Guralnick do show this is true if one considers simple Chevalley groups over fields of bounded size.

\subsection{Fixed point ratios}

Previous sections of this paper have discussed fix $(x)$, the number of fixed points of an element $x$ of $G$ on a set $\Omega$. This subsection concerns the fixed point ratio $r f i x(x)=\frac{f i x(x)}{|\Omega|}$. We describe applications to random generation. For many other applications (base size, Guralnick-Thompson conjecture, etc.), see the survey [65]. It should also be mentioned that fixed point ratios are a special case of character ratios, which have numerous applications to areas such as random walk [23] and number theory [40].

Let $P(G)$ denote the probability that two random elements of a finite group $G$ generate $G$. One of the first results concerning $P(G)$ is due to Dixon [30], who proved that $\lim _{n \rightarrow \infty} P\left(A_{n}\right)=1$. The corresponding result for finite simple classical groups is due to Kantor and Lubotzky [49]. The strategy adopted by Kantor and Lubotzky was to first note that for any pair $g, h \in G$, one has that $\langle g, h\rangle \neq G$ if and only if $\langle g, h\rangle$ is contained in a maximal subgroup $M$ of $G$. Since $P(g, h \in M)=(|M| /|G|)^{2}$, it follows that

$$
1-P(G) \leq \sum_{M}\left(\frac{|M|}{|G|}\right)^{2} \leq \sum_{i}\left(\frac{\left|M_{i}\right|}{|G|}\right)^{2}\left(\frac{|G|}{\left|M_{i}\right|}\right)=\sum_{i} \frac{\left|M_{i}\right|}{|G|} .
$$

Here $M$ denotes a maximal subgroup and $\left\{M_{i}\right\}$ are representatives of conjugacy classes of maximal subgroups. Roughly, to show that this sum is small, one can use Aschbacher's classification of maximal subgroups [4], together with Liebeck's upper bounds on sizes of maximal subgroups [53].

Now suppose that one wants to study $P_{x}(G)$, the chance that a fixed element $x$ and a random element $g$ of $G$ generate $G$. Then

$$
1-P_{x}(G)=P(\langle x, g\rangle \neq G) \leq \sum_{M} P(g \in M)=\sum_{\substack{\text { maximal } \\ x \in M}} \frac{|M|}{|G|}
$$

Here the sum is over maximal subgroups $M$ containing $x$. Let $\left\{M_{i}\right\}$ be a set of representatives of maximal subgroups of $G$, and write $M \sim M_{i}$ if $M$ is conjugate to $M_{i}$. Then the above sum becomes

$$
\sum_{i} \frac{\left|M_{i}\right|}{|G|} \sum_{\substack{M \sim M_{i} \\ x \in M}} 1
$$


To proceed further we assume that $G$ is simple. Then, letting $N_{G}\left(M_{i}\right)$ denote the normalizer of $M_{i}$ in $G$, one has that

$$
g_{1} M_{i} g_{1}^{-1}=g_{2} M_{i} g_{2}^{-1} \leftrightarrow g_{1}^{-1} g_{2} \in N_{G}\left(M_{i}\right) \leftrightarrow g_{1}^{-1} g_{2} \in M_{i} \leftrightarrow g_{1} M_{i}=g_{2} M_{i}
$$

In other words, there is a bijection between conjugates of $M_{i}$ and left cosets of $M_{i}$. Moreover, $x \in g M_{i} g^{-1}$ if and only if $x g M_{i}=g M_{i}$. Thus

$$
\frac{\left|M_{i}\right|}{|G|} \sum_{\substack{M \sim M_{i} \\ x \in M}} 1=\operatorname{rfix}\left(x, M_{i}\right) .
$$

Here $\operatorname{rfix}\left(x, M_{i}\right)$ denotes the fixed point ratio of $x$ on left cosets of $M_{i}$, that is the proportion of left cosets of $M_{i}$ fixed by $x$. Summarizing, $P_{x}(G)$ can be upper bounded in terms of the quantities $r f i x\left(x, M_{i}\right)$. This fact has been usefully applied in quite a few papers (see $[39,43]$ and the references therein, for example).

\subsection{Miscellany}

This subsection collects some miscellaneous facts about fixed points and derangements.

(1) Formulae for fixed points

We next state a well-known elementary proposition which gives different formulae for the number of fixed points of an element in a group action.

Proposition 8.4 Let $G$ be a finite group acting transitively on $X$. Let $C$ be a conjugacy class of $G$ and $g$ in $C$. Let $H$ be the stabilizer of a point in $X$.

(1) The number of fixed points of $g$ on $X$ is $\frac{|C \cap H|}{|C|}|X|$.

(2) The fixed point ratio of $g$ on $X$ is $\frac{|C \cap H|}{|C|}$.

(3) The number of fixed points of $g$ on $X$ is $\left|C_{G}(g)\right| \sum_{i}\left|C_{H}\left(g_{i}\right)\right|^{-1}$ where the $g_{i}$ are representatives for the $\mathrm{H}$ classes of $\mathrm{C} \cap \mathrm{H}$.

Proof Clearly (1) and (2) are equivalent. To prove (1), we determine the cardinality of the set $\{(u, x) \in C \times X \mid u x=x\}$. On the one hand, this set has size $|C| f(g)$ where $f(g)$ is the number of fixed points of $g$. On the other hand, it is $|X||C \cap H|$, whence (1) holds.

For (3), note that $|C|=\frac{|G|}{\left|C_{G}(g)\right|}$ and $|C \cap H|=\sum_{i} \frac{|H|}{\left|C_{H}\left(g_{i}\right)\right|}$ where the $g_{i}$ are representatives for the $H$-classes of $C \cap H$. Plugging this into (1) and using $|X|=\frac{|G|}{|H|}$ completes the proof.

\section{(2) Algorithmic issues}

It is natural to ask for an algorithm to generate a random derangement in $S_{n}$, for example for cryptographic purposes. One such algorithm appears in [21]. Peter Cameron (personal communication) has shown us how to use the recurrence for derangement numbers to produce a second algorithm for random generation. There is 
also a literature on Gray codes for running through all derangements in the symmetric group; see [8] and [50].

\section{(3) Algebraic combinatorics}

The set of derangements has itself been the subject of some combinatorial study. For example, Désarménien [21] has shown that there is a bijection between derangements in $S_{n}$ and the set of permutations with first ascent occurring in an even position. This is extended and refined by Désarménien and Wachs [22]. Diaconis, McGrath, and Pitman [27] study the set of derangements with a single descent. They show that this set admits an associative, commutative product and unique factorization into cyclic elements. Bóna [11] studies the distribution of cycles in derangements, using among other things a result of E. Canfield that the associated generating function has all real zeros. Benkart and Doty [9] relate derangements and tensor powers of adjoint modules for the Lie algebra $g=s l_{n}$. In particular, they show that for $n \geq 2 k$, the centralizer algebra $E n d_{g}\left(s l_{n}^{\otimes k}\right)$ has a basis indexed by the derangements of $S_{2 k}$.

(4) Statistics

The fixed points of a permutation give rise to a useful metric on the permutation group: the Hamming metric. Thus $d(\pi, \sigma)$ is equal to the number of places where $\pi$ and $\sigma$ disagree. This is a bi-invariant metric on the permutation group and

$$
d(\pi, \sigma)=d\left(i d, \pi^{-1} \sigma\right)=\text { number of fixed points in } \pi^{-1} \sigma .
$$

Such metrics have many statistical applications (Chapter 6 of [23]).

\section{References}

1. Aldous, D.: Probability Approximations via the Poisson Clumping Heuristic. Applied Mathematical Sciences, vol. 77. Springer, New York (1989)

2. Arratia, R., Tavaré, S.: The cycle structure of random permutations. Ann. Probab. 20, 1567-1591 (1992)

3. Aschbacher, M.: Finite Group Theory, 2nd edn. Cambridge Studies in Advanced Mathematics, vol. 10. Cambridge University Press, Cambridge (2000)

4. Aschbacher, M.: On the maximal subgroups of finite classical groups. Invent. Math. 76, 469-514 (1984)

5. Aschbacher, M., Scott, L.: Maximal subgroups of finite groups. J. Algebra 92, 44-80 (1985)

6. Babai, L.: On the order of uniprimitive permutation groups. Ann. Math. 113, 553-568 (1981)

7. Barbour, A., Holst, L., Janson, S.: Poisson Approximation. Oxford Science Publications. Clarendon, New York (1992)

8. Baril, J., Vajnovszki, V.: Gray code for derangements. Discrete Appl. Math. 140, 207-221 (2004)

9. Benkart, G., Doty, S.: Derangements and tensor powers of adjoint modules for $s l_{n}$. J. Algebr. Comb. 16, 31-42 (2002)

10. Bidigare, P., Hanlon, P., Rockmore, D.: A combinatorial description of the spectrum of the Tsetlin library and its generalization to hyperplane arrangements. Duke Math. J. 99, 135-174 (1999)

11. Bóna, M.: On a balanced property of derangements. Electron. J. Comb. 13, 12 (2006). Research Paper 102 (electronic)

12. Boston, N., Dabrowski, T., Foguel, P., Gies, P., Leavitt, J., Ose, D., Jackson, D.A.: The proportion of fixed-point-free elements of a transitive permutation group. Commun. Algebra 21, 3259-3275 (1993)

13. Bovey, J.: The probability that some power of a permutation has small degree. Bull. Lond. Math. Soc. 12, 47-51 (1980)

14. Buhler, J., Lenstra, H.W. Jr., Pomerance, C.: Factoring integers with the number field sieve. In: The Development of the Number Field Sieve. Lecture Notes in Mathematics, vol. 1554. Springer, Berlin (1993) 
15. Cameron, P.: Permutation Groups. London Mathematical Society Student Texts, vol. 45. Cambridge University Press, Cambridge (1999)

16. Cameron, P.: Derangements and p-elements in permutation groups. Online lecture notes (2007), available at: http://www.maths.qmul.ac.uk/ pjc/slides

17. Cameron, P., Cohen, A.M.: On the number of fixed point free elements in a permutation group. Discrete Math. 106/107, 135-138 (1992)

18. Chatterjee, S., Diaconis, P., Meckes, E.: Exchangeable pairs and Poisson approximation. Probab. Surv. 2, 64-106 (2005) (electronic)

19. Chowla, S.: The Riemann zeta and allied functions. Bull. Am. Math. Soc. 58, 287-303 (1952)

20. Cohen, S.D.: The distribution of polynomials over finite fields. Acta Arith. 17, 255-271 (1970)

21. Désarménien, J.: Une autre interprétation du nombre de dérangements. Sém. Lothar. Comb. 9, 11-16 (1984)

22. Désarménien, J., Wachs, M.: Descent classes of permutations with a given number of fixed points. J. Comb. Theory Ser. A 64, 311-328 (1993)

23. Diaconis, P.: Group Representations in Probability and Statistics. Institute of Mathematical Statistics Lecture Notes, vol. 11. Hayward (1988)

24. Diaconis, P., Fill, J., Pitman, J.: Analysis of top to random shuffles. Comb. Probab. Comput. 1, 135155 (1992)

25. Diaconis, P., Holmes, S.: Matchings and phylogenetic trees. Proc. Natl. Acad. Sci. USA 95, 1460014602 (1998)

26. Diaconis, P., Holmes, S.: Random walk on trees and matchings. Electron. J. Probab. 7, 1-17 (2002)

27. Diaconis, P., McGrath, M., Pitman, J.: Riffle shuffles, cycles, and descents. Combinatorica 15, 11-29 (1995)

28. Diaconis, P., Shahshahani, M.: On the eigenvalues of random matrices. J. Appl. Probab. A 31, 49-62 (1994)

29. Dixon, J.: Random sets which invariably generate the symmetric group. Discrete Math. 105, 25-39 (1992)

30. Dixon, J.: The probability of generating the symmetric group. Math. Z. 110, 199-205 (1969)

31. Dixon, J., Mortimer, B.: Permutation Groups. Graduate Texts in Mathematics, 163. Springer, Berlin (1996)

32. Durrett, R.: Probability: Theory and Examples, 2nd edn. Duxbury Press (1996)

33. Feller, W.: An Introduction to Probability Theory and Its Applications, vol. 1, 3rd edn. Wiley, New York (1968)

34. Fulman, J.: Card shuffling and the decomposition of tensor products. Pac. J. Math. 217, 247-262 (2004)

35. Fulman, J.: Convergence rates of random walk on irreducible representations of finite groups. J. Theor. Probab. 21, 193-211 (2008)

36. Fulman, J., Guralnick, R.: Derangements in simple and primitive groups. In: Groups, Combinatorics, and Geometry, Durham, 2001, pp. 99-121. World Scientific, Singapore (2003)

37. Fulman, J., Guralnick, R.: Derangements in subspace actions of classical groups. Preprint

38. Fulman, J., Guralnick, R.: Derangements in classical groups for non-subspace actions. Preprint

39. Fulman, J., Guralnick, R.: The probability of generating an irreducible subgroup. Preprint

40. Gluck, D., Magaard, K.: Absolute fourth moments and finiteness of linear groups. Commun. Algebra 34, 3387-3407 (2006)

41. Goncharov, V.: Sur la distribution des cycles dans les permutations. C. R. (Dokl.) Acad. Sci. URSS (N.S.) 35, 267-269 (1942)

42. Guralnick, R.: Monodromy groups of coverings of curves. In: Galois Groups and Fundamental Groups. Math. Sci. Res. Inst. Publ., vol. 41, pp. 1-46. Cambridge Univ. Press, Cambridge (2003)

43. Guralnick, R., Kantor, W.: Probabilistic generation of finite simple groups. J. Algebra 234, 743-792 (2000)

44. Guralnick, R., Kimmerle, W.: On the cohomology of alternating and symmetric groups and decomposition of relation modules. J. Pure Appl. Algebra 69, 135-140 (1990)

45. Guralnick, R., Magaard, K.: On the minimal degree of a primitive permutation group. J. Algebra 207, 127-145 (1998)

46. Guralnick, R., Wan, D.: Bounds for fixed point free elements in a transitive group and applications to curves over finite fields. Isr. J. Math. 101, 255-287 (1997)

47. James, G., Kerber, A.: The Representation Theory of the Symmetric Group. Encyclopedia of Mathematics and its Applications, vol. 16. Addison-Wesley, Reading (1981) 
48. Jordan, C.: Recherches sur les substitutions. J. Liouville 17, 351-367 (1872)

49. Kantor, W., Lubotzky, A.: The probability of generating a finite classical group. Geom. Dedic. 36, 67-87 (1990)

50. Korsh, J., LaFollette, P.: Constant time generation for derangements. Inf. Process. Lett. 90, 181-186 (2004)

51. Kurzweil, H., Stellmacher, B.: The Theory of Finite Groups: an Introduction. Springer, Berlin (2004)

52. Lenstra, H.W. Jr., Stevenhagen, P.: Chebotarev and his density theorem. Math. Intel. 18, 26-37 (1996)

53. Liebeck, M.: On the order of maximal subgroups of finite classical groups. Proc. Lond. Math. Soc. 50, 426-446 (1985)

54. Liebeck, M., Praeger, C., Saxl, J.: A classification of the maximal subgroups of the finite alternating and symmetric groups. J. Algebra 111, 365-383 (1987)

55. Liebeck, M., Praeger, C., Saxl, J.: On the O'Nan-Scott theorem for finite primitive permutation groups. J. Austral. Math. Soc. Ser. A 44, 389-396 (1988)

56. Lovász, L., Plummer, M.: Matching Theory. Annals of Discrete Mathematics, vol. 29. North Holland, Amsterdam (1986)

57. Luczak, T., Pyber, L.: On random generation of the symmetric group. Comb. Probab. Comput. 2, 505-512 (1993)

58. de Montmort, P.R.: Essay d'Analyse sur les Jeux de Hazard, 1st edn. (1708), 2nd edn. (1713). Jacques Quillau, Paris. Reprinted 2005 by AMS/Chelsea, New York

59. Mortimer, B.: Permutation groups containing affine groups of the same degree. J. Lond. Math. Soc. 15, 445-455 (1977)

60. Newton, B., Benesh, B.: A classification of certain maximal subgroups of symmetric groups. J. Algebra 304, 1108-1113 (2006)

61. Odlyzko, A.M.: Asymptotic enumeration methods. In: Handbook of Combinatorics, vol. II. MIT Press/Elsevier, Cambridge (1995)

62. Passman, D.: Permutation Groups. Benjamin, New York (1968)

63. Serre, J.P.: On a theorem of Jordan. Bull. Am. Math. Soc. 40, 429-440 (2003)

64. Shalev, A.: A theorem on random matrices and some applications. J. Algebra 199, 124-141 (1998)

65. Shalev, A.: Probabilistic group theory. In: Groups St. Andrews 1997. Lond. Math. Soc. Lecture Note Ser., vol. 261, pp. 648-678. Cambridge Univ. Press., Cambridge (1999)

66. Shepp, L., Lloyd, S.: Ordered cycle lengths in a random permutation. Trans. Am. Math. Soc. 121, 340-357 (1966)

67. Stanley, R.: Enumerative Combinatorics, vol. 1: Corrected reprint of the 1986 original. Cambridge Studies in Advanced Mathematics, vol. 49. Cambridge University Press, Cambridge (1997)

68. Takács, L.: The problem of coincidences. Arch. Hist. Exact Sci. 21(3), 229-244 (1979) 